\title{
ON IDEALS OF FREE AND FREE NILPOTENT LIE ALGEBRAS
}

\begin{abstract}
MELIH BORAL
ABSTRACT. It is proved that in a free nilpotent Lie algebra there are no nonabelian ideals which are free nilpotent as subalgebras. It is also shown that, for any proper ideal $S$ of a free Lie algebra $F$, the quotient of the lower central terms $F_{m} / S_{m}$ is not finitely generated when $F \neq F_{2}+S$. If $F=F_{2}+S, F / S$ is finite-dimensional and $S$ is finitely generated as an ideal in $F$, then $F_{m} / S_{m}$ is finitely generated as an algebra for all $m \geqslant 1$.
\end{abstract}

1. Introduction. It is known that every subalgebra of a free Lie algebra is again free (Širšov [3]), but a nonabelian ideal, and hence a nonabelian subalgebra of a free nilpotent Lie algebra, is not, in general, free nilpotent. In the first part of this paper we prove that in a free nilpotent Lie algebra there are no proper nonabelian ideals which are free nilpotent as subalgebras.

In the second part we consider the quotient algebra of a lower central term of a free Lie algebra by an ideal and note certain differences with analogous problems in group theory. If $F$ is a free Lie algebra, $S$ any proper ideal of $F$ and $F_{m}, S_{m}$ are the $m$ th terms of the lower central series of $F, S$, respectively, we prove that $F_{m} / S_{m}$ is not finitely generated when $F \neq F_{2}+S$. We will also show that if $F=F_{2}+S, F / S$ is finite-dimensional and $S$ is finitely generated as an ideal in $F$, then $F_{m} / S_{m}$ is finitely generated (as an algebra) for all $m \geqslant 1$.

We consider Lie algebras over a field $\Gamma$. The Lie operation is indicated by juxtaposition and we write

$$
\underbrace{a(a(\cdots(a b)) \cdots)}_{r \text { times }}=a^{r} \cdot b
$$

where $a$ and $b$ are elements of a Lie algebra $L$. We use Greek letters for the elements of the field $\Gamma$. By $L_{m}$ we denote the $m$ th term of the lower central series of $L$ and say that $L$ is free nilpotent of class $n$ if there is a free Lie algebra $F$ of the same rank as $L$ such that $L$ is isomorphic to $F / F_{n+1}$. If $H$ is a Hall basis for the free Lie algebra $F$, then the elements in $H$ of length $\leqslant n$ form a basis for $L$ (Hall [2]). Given any $b \in F$, expressed as a linear combination of these basis elements, by the leading term of $b$, we understand the minimal term of $H$ which appears in that expression (minimal with respect to the length preserving order in $H)$ and denote it by $\operatorname{ld}(b)$.

Received by the editors December 23, 1983 and, in revised form, May 18, 1984. 1980 Mathematics Subject Classification. Primary 17B30, 17B05; Secondary 17B65.

Key' words and phrases. Free Lie algebras, ideals, free nilpotent subalgebras, quotients of lower terms. 
2. Ideals as free nilpotent subalgebras. Every abelian ideal of a free nilpotent Lie algebra is free abelian. A nonabelian ideal, however, is not necessarily free nilpotent as a subalgebra (consider $L_{2}$ as an ideal in $L=F / F_{5}$ ). We have

THEOREM 2.1. The only ideals which are free nilpotent subalgebras in a free nilpotent Lie algebra are free abelian or the whole algebra.

Proof. Let $L$ be a free nilpotent Lie algebra of class $n$ and $X$ a free generating set for $L$. If $|X|=1$ or $L$ is abelian, then there is nothing to prove. Hence we presuppose that $|X|>1$ and $n>1$. Let us also assume that $H$ is a Hall basis for $L$ constructed on $X$, and $S$ is a proper nonabelian ideal which is free nilpotent of class $r>1$, where $r \leqslant n$. We will show that this leads to a contradiction. We consider two cases separately:

Case I. $r<n$. Let $q$ be the integer such that $S \subseteq L_{q}$ but $S \nsubseteq L_{q+1}$. Choose a free generating set $Y$ for $S$, which contains an element $y$ whose leading term has length $q$. Suppose that $q=1$. If $Y$ has at least two elements which are linearly independent modulo $L_{2}$, then we can produce an element of $S_{n}$, which is nonzero in $L$, contradicting $r<n$. Now assume that $Y$ has only one element $y$ which is nonzero modulo $L_{2}$ and $Y-\{y\} \subseteq L_{2}$. Choose $x \in X$ such that $x$ is not equal to a scalar multiple of the leading term of $y$. Then the element $x \cdot{ }^{n-1} y \in S$ belongs to $L_{n}$, but due to the form of its leading term it cannot be written as a product of two elements of $S$, and hence $x \cdot{ }^{n-1} y \notin S_{2}$. Thus the element $y\left(x \cdot{ }^{n-1} y\right) \in S_{2}$ does not belong to $S_{3}$ but it belongs to $L_{n+1}$ and hence it is trivial in $L$, contradicting $r>1$.

Suppose now that $q>1$. Now for any $x \in X, x y \in S$ and its leading term has length $q+1$. Hence $x y \notin S_{2}$ since any nonzero element of $S_{2}$ has leading term of length $\geqslant 2 q$. Then the element $t=y \cdot{ }^{r}(x y)$ in $S_{r+1}$ has leading term of length $q r+(q+1)$. But $S_{r+1} \subseteq L_{n+1}$ implies that $q r+(q+1) \geqslant n+1$ or, equivalently,

$$
(r+1) q \geqslant n .
$$

Now for some $x^{\prime} \neq x$ in $X$, consider the elements $f=x \cdot \cdot^{q-2}(x y)$ and $g=x^{\prime} \cdot{ }^{q-2}(x y)$ of $S$. Both have leading terms with lengths $(q-2)+(q+1)=2 q-1$ and hence $f, g \notin S_{2}$. Then the element $w=f^{r-1} g \notin S_{r+1}$ implies that the length of $\operatorname{ld}(w)=$ $r(2 q-1)<n+1$. Equivalently,

$$
(r+1) q+(r q-q-r)<n+1 .
$$

Comparing with (1) we obtain the inequality $r q-q-r \leqslant 0$. Therefore

$$
(r-1) q \leqslant r \text {. }
$$

Since $q>1$ we have $q=2$ and $r=2$ as the only possibility left for consideration, in which case, $S \subseteq L_{2}$ but $S \nsubseteq L_{3}$ and $S_{3} \subseteq L_{n+1}$. Then $Y$ contains $y=\beta(a b)+\cdots$, where $0 \neq \beta \in \Gamma$ and $a b$ is its leading term, $a, b \in X$. Let

$$
u=a y=\beta a(a b)+\cdots, \quad v=a(a y)=\beta a(a(a b))+\cdots,
$$

both elements in $S$. Due to the nature of their leading terms they are not expressible as linear combinations of products of elements of $Y$ and hence $u, v \notin S_{2}$. Then $u v \notin S_{3}$, which implies that length of $\operatorname{ld}(u v)=7<n+1$. But $y(y u) \in S_{3}$ implies that length of $\operatorname{ld}(y(y u))=7 \geqslant n+1$. Therefore

$$
7<n+1 \leqslant 7 \text {, }
$$

which is a contradiction. 
Case II. $r=n$. Let $Y=\left\{y_{1}, y_{2}, \ldots\right\}$ be a free generating set for $S$. Since $r=n, Y$ is linearly independent modulo $L_{2}$. Let $x \in L-S, x \notin L_{2}, T$ be the subalgebra of $L$ generated by $x$ and $S$, and $H^{Y}$ be a Hall basis for $S$ on $Y$. The set $H^{Y} \cup\{x\}$ is linearly independent in $L$ or else we contradict $x \notin \mathrm{S}$. Furthermore, any element in $T$ can be expressed as a linear combination of elements in $H^{Y} \cup\{x\}$, which implies that it is a basis for $T$ and that $T$ is generated by the set $Y \cup\{x\}$.

Suppose that $Y \cup\{x\}$ is linearly dependent modulo $L_{2}$. Then

$$
x=a+f
$$

where $a=\sum_{i} \alpha_{i} y_{i} \notin L_{2}, \alpha_{i} \in \Gamma$ and $f \in L_{2}$. Multiplying (2) by $x, n-1$ times, we obtain $x \cdot{ }^{n-1} a=-x \cdot{ }^{n-1} f \in L_{n+1}$. But $x \cdot{ }^{n-1} a \notin L_{n+1}$ is a nonzero element of $L$, a contradiction. Thus $\{x\} \cup Y$ is linearly independent modulo $L_{2}$. Then it freely generates $T$. Let $x<y_{1}<y_{2}<\cdots$ be an ordering on $\{x\} \cup Y$ and $H^{\prime}$ be a Hall basis constructed on this set such that $H^{\prime} \supseteq H^{Y}$. Then the element $x y_{1}$ of $H^{\prime}$ belongs to $S$ and $x y_{1} \in S_{2}$ since $S$ is free nilpotent of class $r=n$. Then

$$
x y_{1}=\sum_{i<j} \gamma_{i j} y_{i} y_{j}+f,
$$

where $\gamma_{i j} \in \Gamma$ (not all zero), $f \in S_{3}$ and $y_{i} y_{j} \in H^{Y} \subseteq H^{\prime}$. But (3) contradicts the linear independence of elements of $H^{\prime}$ modulo $T_{3}$. Q.E.D.

3. The quotient algebra of a lower central term of a Lie algebra by an ideal.

D. Spellman [4] studied the relationship of the lower central series of a normal subgroup of finite index to the lower central series of a free group of finite rank. We consider similar problems for ideals in general of free Lie algebras. Our assumptions, methods and results will differ from those of [4].

It is proved by B. Baumslag [1] that a nonzero finitely generated subalgebra which is an ideal in a free Lie algebra must be the whole algebra. Let $F$ be a free Lie algebra, $S$ an ideal of $F$ and consider the nontrivial case where $S$ is not finitely generated as a subalgebra. Clearly $S_{m}$ is an ideal of $F_{m}$ and we can form $F_{m} / S_{m}$. The terms of the lower central series $F_{m}$ are not finitely generated when $F$ is generated by more than one element.

In a free group $G$ of finite rank, there are certain proper normal subgroups $N$ such that $G_{m} / N_{m}$ is an infinite group but it is of finite rank. For Lie algebras as well, there are cases where $F_{m} / S_{m}$ is finitely generated. We first prove

Proposition 3.1. Let $S$ be a proper ideal in a free Lie algebra $F$ such that $F \neq F_{2}+S$. Then $F_{m} / S_{m}$ is not finitely generated for $m>1$.

Proof. To prove that $F_{m} / S_{m}, m>1$, is not finitely generated, it is sufficient to show that $\left(F_{m} / S_{m}\right) /\left(F_{m} / S_{m}\right)_{2}=F_{m} /\left(\left(F_{m}\right)_{2}+S_{m}\right)$ is infinite-dimensional. Replacing $S$ by the proper ideal $S+F_{2}$ we see that we may assume $S \supseteq F_{2}$. Thus we may choose a free generating set $\{a, b, c, \ldots\}$ for $F$ such that $a \notin S, S \subseteq F_{2}+$ $\operatorname{span}\{b, c, \ldots\}$. Give $F$ a grading by setting $\operatorname{deg}(a)=A$ and $\operatorname{deg}(x)=B$ for all the other generators $b, c, \ldots$ Then

$$
F_{m} \subseteq F_{2} \subseteq \sum_{j>0} F^{(i A+j B)}
$$


so $\left(F_{m}\right)_{2} \subseteq \sum_{j>1} F^{(i A+j B)}$. But $S \subseteq \sum_{j>0} F^{(i A+j B)}$ implies that $S_{m} \subseteq S_{2} \subseteq$ $\sum_{j>1} F^{(i A+j B)}$. Thus

$$
\left(F_{m}\right)_{2}+S_{m} \subseteq \sum_{j>1} F^{(i A+j B)} .
$$

If $C=\left\{a \cdot{ }^{i} b: i \geqslant m-1\right\}$, then $C$ is an infinite linearly independent set contained in $\sum F^{(i A+B)}$. Since

$$
\left(\sum F^{(i A+B)}\right) \cap\left(\sum_{j>1} F^{(i A+j B)}\right)=\{0\},
$$

we conclude that the image of $C$ in $F_{m} /\left(\left(F_{m}\right)_{2}+S_{m}\right)$ is linearly independent. Hence $F_{m} / S_{m}$ is not finitely generated.

If $F=F_{2}+S$, then $F_{m} / S_{m}$ may be finitely generated. We first consider the case where $m=2$.

Proposition 3.2. Let $S$ be a proper ideal in a free Lie algebra $F$. Suppose that $F=F_{2}+S, F / S$ is finite-dimensional and $S$ is finitely generated as an ideal in $F$. Then $F_{2} / S_{2}$ is finitely generated as an algebra.

Proof. $F_{2}$ contains the ideal $F_{2} \cap S$, and so in $F_{2} / S_{2}$ we have

$$
F_{2} / S_{2} \supseteq\left(F_{2} \cap S\right) / S_{2} \text {, }
$$

where the second term is an abelian ideal. The quotient $\left(F_{2} / S_{2}\right) /\left(\left(F_{2} \cap S\right) / S_{2}\right) \cong$ $F_{2} /\left(F_{2} \cap S\right) \cong\left(F_{2}+S\right) / S=F / S$ is finite-dimensional, and so it suffices to show that $\left(F_{2} \cap S\right) / S_{2}$ is finitely generated as an $F / S$-module.

Let $\left\{s_{i}: i \in I\right\}$ be a finite generating set for $S$ as an $F$-module. This generating set may be chosen so that there is some subset $I^{\prime} \subseteq I$ such that $F_{2} \cap \operatorname{span}\left\{s_{i}: i \in I\right\}=$ $\operatorname{span}\left\{s_{i}: i \in I^{\prime}\right\}$.

Let $\left\{w_{j}: j \in J\right\}$ be a basis for $F / S$. As $F / S$ is finite-dimensional $J$ is finite. Now as $\left\{s_{i}: i \in I\right\}$ generates $S$ as an $F$-module, $\left\{s_{i}+S_{2}: i \in I\right\}$ generates $S / S_{2}$ as an $F / S$-module. Thus (by the Poincaré-Birkhoff-Witt Theorem) $S / S_{2}$ is generated by the set

$$
\left\{\left(\prod_{j \in J} w_{j}^{n_{j}}\right) s_{i}: i \in I, n_{j} \geqslant 0\right\},
$$

where the product $\prod_{j \in J} w_{j}^{n_{j}}$ is taken in some fixed order. Clearly $F_{2} \cap S / S_{2}$ is spanned by

$$
\left\{\left(\prod_{j \in J} w_{j}^{n_{j}}\right) s_{i}: i \in I, n_{j} \geqslant 0, \sum n_{j}>0\right\} \cup\left\{s_{i}: i \in I^{\prime}\right\},
$$

and so $F_{2} \cap S / S_{2}$ is generated as an $F / S$-module by the finite set $\left\{w_{j} s_{i}: i \in I\right.$, $j \in J\} \cup\left\{s_{i}: i \in I^{\prime}\right\}$. This proves the proposition.

Let us note that it is possible to satisfy all the requirements in the hypothesis above. As an example take $F$ to be the free Lie algebra on $\{e, f, h\}$ and $S$ to be generated by $(e f)-h$, $(h e)-2 e$, and $(h f)+2 f$. Then $F=F_{2}+S$ and all the conditions are satisfied. 
To prove a similar result for the case $m>2$, we first need

LEMMA 3.3. If $S$ is a proper ideal of a free Lie algebra $F$ such that $F=F_{2}+S$, then $F_{m-1}=F_{m}+S_{m-1}$ for all $m \geqslant 2$.

Proof. The result holds for $m=2$ by hypothesis. Assuming the result to be true for $m-1$ we see that

$$
F_{m-1}=\left[F, F_{m-2}\right]=\left[F_{2}+S, F_{m-1}+S_{m-2}\right] \subseteq F_{m}+S_{m-1},
$$

and so the result holds by induction. (Note that if $H$ and $K$ are subspaces of $F$, $[H, K]$ is the subspace spanned by all elements of the form $(h k)$, where $h \in H$ and $k \in K$.)

Proposition 3.4. Let $S$ be a proper ideal in a free Lie algebra $F$. Suppose that $F=F_{2}+S, F / S$ is finite-dimensional and $S$ is finitely generated as an ideal in $F$. Then $F_{m} / S_{m}$ is finitely generated (as an algebra) for all $m \geqslant 1$.

Proof. We proceed by induction on $m$. The case $m=1$ is obvious and the case $m=2$ is done above. Thus we assume that $m>2$.

We assume that $F_{m-1} / S_{m-1}$ is finitely generated. $F_{m}$ contains the ideal $F_{m} \cap S_{m-1}$ and

$$
F_{m} /\left(F_{m} \cap S_{m-1}\right) \cong\left(F_{m}+S_{m-1}\right) / S_{m-1} \cong F_{m-1} / S_{m-1},
$$

so $F_{m} /\left(F_{m} \cap S_{m-1}\right)$ is finitely generated.

For any set $X \subseteq F$, let $G(X)$ denote the subalgebra of $F$ generated by $X$. Then $F_{m}$ contains a finite subset $X$ such that $F_{m}=G(X)+\left(F_{m} \cap S_{m-1}\right)$. Therefore $F_{m} \cap S_{i}$ $=\left(G(X)+\left(F_{m} \cap S_{m-1}\right)\right)$, and $\left(F_{m} \cap S_{i}\right)=\left(G(X) \cap S_{i}\right)+\left(F_{m} \cap S_{m-1}\right)$ for $1 \leqslant i$ $\leqslant m-2$, and so

$$
\begin{aligned}
{\left[F_{m}\right.} & \left.\cap S_{i}, F_{m} \cap S_{m-i-1}\right] \\
& \subseteq\left[\left(G(X) \cap S_{i}\right)+\left(F_{m} \cap S_{m-1}\right),\left(G(X) \cap S_{m-i-1}\right)+\left(F_{m} \cap S_{m-1}\right)\right] \\
& \subseteq G(X)+S_{m} .
\end{aligned}
$$

Therefore, letting $I=\sum_{i=1}^{i=m-2}\left[F_{m} \cap S_{i}, F_{m} \cap S_{m-i-1}\right]$, it is sufficient to show that $\left(F_{m} \cap S_{m-1}\right) / S_{m}+I$ is finitely generated as an $F_{m} /\left(F_{m} \cap S\right) \cong\left(F_{m}+S\right) / S=$ $F / S$-module.

We first show that $S_{m-1} / S_{m}+I$ is finitely generated as an $F / S$-module. Let $K$ be a finite generating set for $S$ as an ideal of $F$ and let

$$
F^{i} K=\operatorname{span}\left\{\left(\operatorname{ad} x_{1}\right) \cdots\left(\operatorname{ad} x_{i}\right) k: x_{1}, \ldots, x_{i} \in F, k \in K\right\} .
$$

If $\mathbf{i}=\left(i_{1}, \ldots, i_{m-1}\right)$ let $|\mathbf{i}|=i_{1}+\cdots+i_{m-1}$. Suppose $a, b$ are chosen so that $i_{a} \geqslant i_{b}$ and $i_{b} \geqslant i_{j}$ for all $j \neq a$. (That is, $i_{a}, i_{b}$ are the two largest elements of $\left\{i_{1}, \ldots, i_{m-1}\right\}$.) Let $\|\mathbf{i}\|=i_{a}-i_{b}$ and $Q(\mathbf{i})$ denote the ideal in $F$ generated by

$$
\left[\cdots\left[\left[F^{i_{1}} K, F^{i_{2}} K\right] F^{i_{3}} K\right] \cdots F^{i_{m-1}} K\right] .
$$

The Jacobi identity implies that

$$
\begin{aligned}
Q\left(i_{1}, \ldots, i_{a}, \ldots, i_{m-1}\right) \subseteq & Q\left(i_{1}, \ldots, i_{a}-1, \ldots, i_{m-1}\right) \\
& +\sum_{\substack{j=1 \\
j \neq a}}^{j=m-1} Q\left(i_{1}, \ldots, i_{j}+1, \ldots, i_{a}-1, \ldots, i_{m-1}\right),
\end{aligned}
$$


and thus if $\|\mathbf{i}\|>1$ we have

$$
Q(\mathbf{i}) \subseteq \sum_{\|\mathbf{k}\|<\|\mathbf{i}\|} Q(\mathbf{k}) .
$$

Thus $S_{m-1} \subseteq \Sigma_{\mathbf{i}} Q(\mathbf{i}) \subseteq \Sigma_{\mathbf{i},\|\mathbf{i}\| \leqslant 1} Q(\mathbf{i})$. Now if $\|\mathbf{i}\| \leqslant 1$ and $|\mathbf{i}|>(m-1)^{2}+1$ we see that $i_{a}, i_{b} \geqslant m$. Let $c=\max \{a, b\}$.Then

$$
\begin{aligned}
& {\left.\left[\cdots\left[\left[F^{i_{1}} K, F^{i_{2}} K\right] \cdots F^{i_{c-1}} K\right] F^{i_{c}} K\right] \cdots F^{i_{m-1}} K\right] } \\
& \subseteq\left[\cdots\left[\left[F_{m} \cap S_{c-1}, F_{m} \cap S\right] F^{i_{c+1}} K\right] \cdots F^{i_{m-1}} K\right] \\
& \subseteq(\operatorname{ad} S)^{m-1-c}\left[F_{m} \cap S_{c-1}, F_{m} \cap S\right] \subseteq I
\end{aligned}
$$

and so $Q(i) \subseteq I$. Thus

$$
S_{m-1} \subseteq\left(\sum_{\substack{\mathbf{i} \\\|\mathbf{i}\| \leqslant 1 \\|\mathrm{i}| \leqslant(m-1)^{2}+1}} Q(\mathbf{i})\right)+S_{m}+I .
$$

Since there are only finitely many $\mathbf{i}$ satisfying $\|\mathbf{i}\| \leqslant 1,|\mathbf{i}| \leqslant(m-1)^{2}+1$, and since each $Q(\mathbf{i})+S_{m} / S_{m}$ is finitely generated as an $F / S$-module (as $F / S$ is finite-dimensional), $S_{m-1} / S_{m}+I$ is finitely generated as an $F / S$-module.

Finally, let $\left\{y_{t}: t \in T\right\}$ be a finite generating set for $S_{m-1} / S_{m}+I$ (as an $F / S$-module). This generating set may be so chosen that there is a subset $T^{\prime} \subseteq T$ such that

$$
\operatorname{span}\left\{y_{t}: t \in T^{\prime}\right\}=\left(F_{m} / S_{m}+I\right) \cap \operatorname{span}\left\{y_{t}: t \in T\right\} .
$$

If $\left\{w_{j}: j \in J\right\}$ is a basis for $F / S$, then

$$
\left\{y_{t}: t \in T^{\prime}\right\} \cup\left\{w_{j} y_{t}: j \in J, t \in T\right\}
$$

generates $F_{m} \cap S_{m-1} / S_{m}+I$. This completes the proof of the proposition.

ACKNOWLEDGEMENT. The author wishes to express his thanks to the referee for suggesting certain changes and providing the proofs of Propositions 3.1, 3.2 and 3.4.

\section{REFERENCES}

1. B. Baumslag, Free Lie algebras and free groups, J. London Math. Soc. (2) 4, (1972), 523-532.

2. M. Hall, $A$ basis for free Lie rings and higher commutators in free groups, Proc. Amer. Math. Soc. 1 (1950), 575-581.

3. A. I. Širšov, Subalgebras of free Lie algebras, Mat. Sb. 33(75) (1953), 441-452.

4. D. Spellman, On the respective terms of the lower central series of a free group and a normal subgroup, J. Algebra 31 (1974), 293-304.

Çukurova Üniversitesi, Fen - Edebiyat FakUltesi, Matematik BolumU, P. K. 171, Adana, TURKEY 